\title{
A SYMPLECTIC BANACH SPACE WITH NO LAGRANGIAN SUBSPACES
}

BY

\author{
N. J. KALTON ${ }^{1}$ AND R. C. SWANSON
}

\begin{abstract}
In this paper we construct a symplectic Banach space $(X, \Omega)$ which does not split as a direct sum of closed isotropic subspaces. Thus, the question of whether every symplectic Banach space is isomorphic to one of the canonical form $Y \times Y^{*}$ is settled in the negative. The proof also shows that $\mathcal{L}(X)$ admits a nontrivial continuous homomorphism into $\mathcal{L}(H)$ where $H$ is a Hilbert space.
\end{abstract}

1. Introduction. Given a Banach space $E$, a linear symplectic form on $E$ is a continuous bilinear map $\Omega: E \times E \rightarrow \mathbf{R}$ which is alternating and nondegenerate in the (strong) sense that the induced map $\tilde{\Omega}: E \rightarrow E^{*}$ given by $\tilde{\Omega}(e)(f)=\Omega(e, f)$ is an isomorphism of $E$ onto $E^{*}$. A Banach space with such a form is called a symplectic Banach space. It can be shown, by essentially the argument of Lemma 2 below, that any symplectic Banach space can be renormed so that $\tilde{\Omega}$ is an isometry. Any symplectic Banach space is reflexive.

Standard examples of symplectic Banach spaces all arise in the following way. Let $Y$ be a reflexive Banach space and set $E=Y \oplus Y^{*}$. Define the linear symplectic form $\Omega_{Y}$ by

$$
\Omega_{Y}\left[\left(y, y^{*}\right),\left(z, z^{*}\right)\right]=z^{*}(y)-y^{*}(z) .
$$

We define two symplectic spaces $\left(E_{1}, \Omega_{1}\right)$ and $\left(E_{2}, \Omega_{2}\right)$ to be equivalent if there is an isomorphism $A: E_{1} \rightarrow E_{2}$ such that $\Omega_{2}(A x, A y)=\Omega_{1}(x, y)$.

A. Weinstein [10] has asked the question whether every symplectic Banach space is equivalent to one of the form $\left(Y \oplus Y^{*}, \Omega_{Y}\right)$. (See also [8].)

A closed subspace $F$ of a symplectic space $E$ is isotropic if $\Omega(x, y)=0$ for every $x$, $y \in F . F$ is Lagrangian if it is isotropic and possesses an isotropic complement. As Weinstein notes [10] the question is thus whether every symplectic space has a Lagrangian subspace.

An obvious approach to this question is to construct a maximal isotropic subspace of any given symplectic space. However a maximal isotropic subspace of a symplectic space need not be Lagrangian. Indeed, let $Y$ be a reflexive Banach space and

Received by the editors March 3, 1981 and, in revised form, September 22, 1981.

1980 Mathematics Subject Classification. Primary 58B99, 46B20; Secondary 46D05, 46K 99.

Key words and phrases. Symplectic Banach space, isotropic, Lagrangian subspace, strictly singular operators, $C^{*}$-algebras.

${ }^{1}$ Research partially supported by NSF grant MCS- 8001852 . 
$M$ be an uncomplemented closed subspace of $Y$. Letting $M^{\perp}=\left\{y^{*} \in Y^{*}: y^{*} \mid M=\right.$ $0\}$, the subspace $M \oplus M^{\perp}$ of $\left(Y \oplus Y^{*}, \Omega_{Y}\right)$ is maximally isotropic, but cannot be complemented.

In this paper we shall describe an example of a symplectic space $\left(Z_{2}, \Omega\right)$ which has no Lagrangian subspaces; in fact every complemented isotropic subspace is finite-dimensional. Prior to giving our construction, we shall make some general remarks on the background and motivation behind this problem.

In recent years, various authors have called attention to the practical and theoretical importance of symplectic forms arising in the setting of Banach spaces and, more broadly, Banach manifolds. For example, Weinstein's proof of the Darboux theorem [10] in Banach spaces reduces the local classification of symplectic structures to the study of alternating bilinear forms on Banach spaces. Moreover, symplectic forms in Banach spaces play a decisive rôle in the Hamiltonian formulation of infinite-dimensional mechanics due to P. Chernoff and J. Marsden [2]. Such familiar systems as the Schrodinger equation, the Euler equation of hydrodynamics, the Einstein equations of relativity-all readily lend themselves to a symplectic, i.e. Hamiltonian, approach in Banach spaces. For equations with side conditions, one would employ symplectic forms in Banach manifolds.

Since Lagrangian submanifolds (and their linearizations, Lagrangian subspaces) are preserved by Hamiltonian flows (linear Hamiltonian flows) in Banach spaces, they are natural objects of study. Indeed, a linear Hamiltonian flow always induces a flow of Lagrangian subspaces whose topological features, e.g. intersection number, yields qualitative information about solutions. This point of view was evolved by $\mathrm{J}$. Duistermaat [3] to give an ingenious wholly finite-dimensional proof of the Morse index theorem for geodesics. In Banach spaces, a similar approach leads to index theorems for elliptic partial differential equations with a one-parameter family of boundary values [Swanson, 9].

How much freedom is there in selecting the underlying symplectic Banach space? As Lagrangian subspaces play a strong rôle in applications, are they inevitable given a symplectic Banach space (as in Hilbert space)? This is precisely Weinstein's question given above. The symplectic space $\left(Z_{2}, \Omega\right)$ (that we construct in $\S 2$ ) reveals limits on the amount of structure inherent in the symplectic form itself.

The space $Z_{2}$ was introduced in [6] as a "twisted sum" of two Hilbert spaces; thus $Z_{2}$ has a closed subspace $M \cong l_{2}$ so that $Z_{2} \mid M \cong l_{2}$. It may be noted that all the twisted sums $l_{2, \phi}$ of [6] are symplectic Banach spaces. However $Z_{2}$ has many special properties. It fails to have an unconditional basis and, more generally, fails to have local unconditional structure [5], but has a symmetric unconditional decomposition into two-dimensional subspaces. At the time of this writing, it is unknown whether $Z_{2}$ is prime or isomorphic to each of its closed hyperplanes.

This paper leans heavily on ideas in [6]. However we do point out one deviation from the approach in [6]. In showing that $Z_{2}$ is a Banach space, we use the linear symplectic form $\Omega$ to introduce an equivalent norm, rather than appealing to more general results on type as in [6]. Also in places we indicate simpler arguments than given in [6]. 
We feel that our approach to proving that $Z_{2}$ has no Lagrangian subspaces may have some interest in Banach algebra theory. In particular, we use the symplectic form on $Z_{2}$ to induce an involution on the algebra $L\left(Z_{2}\right)$ of endomorphisms of $Z_{2}$ in the obvious way such that for all $x, y$ in $Z_{2}$ and $T$ in $\mathcal{L}\left(Z_{2}\right), \Omega(T x, y)=\Omega\left(x, T^{*} y\right)$.

Thus, $\mathscr{L}\left(Z_{2}\right)$ is a ${ }^{*}$-algebra. It is easy to see that a projection $P$ on $Z_{2}$ has isotropic range iff $P^{*} P=0$. Indeed, the range is a Lagrangian subspace iff $P^{*} P=0$ and $P^{*}+P=I$. We show that this is not possible by constructing a nontrivial *-homomorphism $\Lambda: \mathcal{L}\left(Z_{2}\right) \rightarrow \mathcal{L}(H)$ where $H$ is a real Hilbert space. In fact, the kernel of $\Lambda$ is the two-sided ideal of strictly singular operators on $Z_{2}$ so that any projection with isotropic range has finite rank.

The example is easily complexified and can be used to solve a problem of Lance [7] on general sesquilinear forms on Banach spaces. However Lance's question as stated requires no reflexivity and has the easy negative solution $E=l_{1}$ as suggested in [7]; the requirement of reflexivity makes a solution much more difficult.

Before constructing the desired example, we shall recall some definitions. A linear mapping of Banach spaces $A: X \rightarrow Y$ is called strictly singular if there is no infinite-dimensional subspace $X_{1} \subset X$ such that $A \mid X_{1}$ is an isomorphism into $Y$.

If $\left(b_{n}\right)$ is a basis of a Banach space $X$ then a block basic sequence of $\left(b_{n}\right)$ is a sequence of form

$$
u_{n}=\sum_{p_{n-1}+1}^{p_{n}} a_{i} b_{i}
$$

where $p_{0}=0<p_{1}<p_{2}<p_{3}<\cdots$.

We shall use repeatedly the "gliding hump" technique. If $v_{n} \in X$ is a sequence such that $v_{n} \rightarrow 0$ weakly, then given any sequence of positive numbers $\varepsilon_{n}$, there is a block basic sequence $\left(u_{n}\right)$ and a subsequence $\left(v_{m_{n}}\right)$ of $\left(v_{n}\right)$ such that $\left\|v_{m_{n}}-u_{n}\right\| \leqslant \varepsilon_{n}$.

2. The example. For any real sequence $x=\left(x_{n}\right)_{n=1}^{\infty}$ we denote by $E x$, the even subsequence of $x$, i.e. Ex $=y$ where $y_{n}=x_{2 n}$.

If $x \in l_{2}$ we denote by $\phi(x)$ the sequence $\left(\phi_{n}(x)\right)_{n=1}^{\infty}$ where

$$
\begin{gathered}
\phi_{2 n}(x)=x_{n}, \quad n=1,2, \ldots, \\
\phi_{2 n-1}(x)=x_{n} \log \frac{\|x\|_{2}}{\left|x_{n}\right|}, \quad n=1,2, \ldots
\end{gathered}
$$

Here, of course, $\|x\|_{2}=\sqrt{\left(\sum\left|x_{n}\right|^{2}\right)}$, and we interpret both $0(\log 0)$ and $0(\log \infty)$ as 0 .

The properties of $\phi$ that we require are essentially established in [6], i.e.

$$
\begin{gathered}
\phi(\alpha x)=\alpha \phi(x), \quad \alpha \in \mathbf{R}, x \in l_{2}, \\
\|\phi(x+y)-\phi(x)-\phi(y)\|_{2} \leqslant C\left(\|x\|_{2}+\|y\|_{2}\right), \quad x, y \in l_{2},
\end{gathered}
$$

where $C$ is a constant independent of $x, y(C=3 \log 2$ will suffice).

Now we define $Z_{2}$ to be the space of sequences $x=\left(x_{n}\right)$ such that

$$
\|x\|=\|E x\|_{2}+\|x-\phi(E x)\|_{2}<\infty .
$$

Then $Z_{2}$ is a linear space and $\|\cdot\|$ is a quasi-norm on $Z_{2}$; in fact

$$
\|x+y\| \leqslant(C+1)(\|x\|+\|y\|) \text {. }
$$


It can be checked that $Z_{2}$ is complete for this quasi-norm and that the standard unit vectors $\left(e_{n}\right)_{n=1}^{\infty}$ form a basis for $Z_{2}$.

The subsequence $\left(e_{1}, e_{3}, \ldots\right)$ spans a closed subspace $M$ of $Z_{2}$ isomorphic to $l_{2}$; in fact if $x \in M,\|x\|=\|x\|_{2}$. It also is easily checked that for any $x \in Z_{2} d(x, M)=$ $\inf _{m \in M}\|x-m\|=\|\mathrm{E} x\|_{2}$ so that the map $E: Z_{2} \rightarrow l_{2}$ is a quotient mapping (i.e. $\left.Z_{2} / M \cong l_{2}\right)$.

We can define an alternating bilinear form $\Omega$ on $\mathbf{R}^{\infty}$ (the finitely nonzero sequences) by

$$
\Omega(x, y)=\sum_{n=1}^{\infty}\left(x_{2 n} y_{2 n-1}-x_{2 n-1} y_{2 n}\right) .
$$

We shall show that $\Omega$ is continuous on $\mathbf{R}^{\infty}$ and hence can be extended to an alternating bilinear form on $Z_{2}$, also denoted by $\Omega$.

LemMa 1 (CF. [6, TheOREM 5.1]). For $x, y \in \mathbf{R}^{\infty},|\Omega(x, y)| \leqslant\|x\|\|y\|$.

Proof. First we claim that

$$
|\Omega(\phi(E x), \phi(E y))| \leqslant \frac{2}{e}\|E x\|_{2}\|E y\|_{2} .
$$

Indeed suppose $\|E x\|_{2}=\|E y\|_{2}=1$. Then

$$
|\Omega(\phi(E x), \phi(E y))|=\left|\sum_{n=1}^{\infty} x_{2 n} y_{2 n} \log \right| \frac{x_{2 n}}{y_{2 n}}|| \leqslant \frac{2}{e},
$$

as in Lemma 5.1 of [5].

Next observe

$$
\begin{gathered}
|\Omega(x-\phi(E x), y)| \leqslant\|x-\phi(E x)\|_{2}\|E y\|_{2}, \\
|\Omega(x, y-\phi(E y))| \leqslant\|E x\|_{2}\|y-\phi(E y)\|_{2}, \\
\Omega(x-\phi(E x), y-\phi(E y))=0 .
\end{gathered}
$$

LEMMA 2. $Z_{2}$ can be given an equivalent norm $\|\cdot\|_{0}$ so that

$$
\|x\|_{0}=\sup _{\|y\|_{0} \leqslant 1}|\Omega(x, y)| \text {. }
$$

Proof. First let

$$
\|x\|=\sup _{\|y\| \leqslant 1}|\Omega(x, y)| .
$$

Then for $x \in M,\|x\|=\|x\|$ while for any $x \in Z_{2}$ we have $\|x\| \geqslant\|E x\|_{2}=$ $d(x, M)$. Thus if $\left\|x^{(n)}\right\| \| \rightarrow 0$ there exist $u^{(n)} \in M$ with $\left\|x^{(n)}-u^{(n)}\right\| \rightarrow 0$. Then $\left\|u^{(n)}\right\| \rightarrow 0$ and so $\left\|x^{(n)}\right\| \rightarrow 0$. It follows that $\|\cdot\|$ is a norm equivalent to the quasi-norm $\|\cdot\|$. Thus there is a constant $B$ so that

$$
\|x\| \leqslant\|x\| \leqslant B\|x\|, \quad x \in Z_{2} .
$$

Now use Zorn's Lemma to choose a minimal norm $\|\cdot\|_{0}$ so that

$$
B^{-1}\|x\| \leqslant\|x\|_{0} \leqslant B\|x\|, \quad x \in Z_{2},
$$

and $|\Omega(x, y)| \leqslant\|x\|_{0}\|y\|_{0}, x, y \in Z_{2}$. Define

$$
\|x\|_{0}^{*}=\sup _{\|y\|_{0} \leqslant 1}|\Omega(x, y)| .
$$


Then

$$
\|x\|_{0}^{*} \geqslant \sup _{B\|y\| \leqslant 1}|\Omega(x, y)| \geqslant \sup _{\|y\| \leqslant B^{-1}}|\Omega(x, y)|=B^{-1}\|x\| \| .
$$

Thus $B^{-1}\|x\| \leqslant\|x\|_{0}^{*} \leqslant\|x\|_{0} \leqslant B\|x\|$. Let

$$
\|x\|_{0}^{* *}=\left(\frac{1}{2}\left(\|x\|_{0}^{*}\right)^{2}+\frac{1}{2}\|x\|_{0}^{2}\right)^{1 / 2}
$$

Then as $|\Omega(x, y)| \leqslant\|x\|_{0}^{*}\|y\|_{0}$ and $|\Omega(x, y)| \leqslant\|x\|_{0}\|y\|_{0}^{*}$, we have

$$
|\Omega(x, y)|^{2} \leqslant \frac{1}{2}\left[\left(\left\|x_{0}\right\|^{*}\left\|x_{0}\right\|\right)^{2}+\left(\|y\|_{0}\|y\|_{0}^{*}\right)^{2}\right]
$$

and hence

$$
|\Omega(x, y)| \leqslant\|x\|_{0}^{* *}\|y\|_{0}^{* *} .
$$

Thus $\|x\|_{0}^{* *}=\|x\|_{0}$ and so $\|x\|_{0}=\left\|x_{0}\right\|^{*}$ has the desired properties.

Although $Z_{2}$ is therefore a Banach space, it is convenient to continue working with the original quasi-norm. Since $Z_{2}$ is reflexive (both $M$ and $Z_{2} / M$ are reflexive), $\Omega$ is a linear symplectic form on $Z_{2}$.

The basis $\left(e_{n}\right)$ is not unconditional. However it is easily seen that if $\varepsilon_{n}= \pm 1$ for all $n$, then the map defined by $S e_{2 n}=\varepsilon_{n} e_{2 n}, S e_{2 n-1}=\varepsilon_{n} e_{2 n-1}$ is an isometry on $Z_{2}$. Thus for some constant $C_{1}$ the operator defined by

$$
\begin{aligned}
S e_{2 n} & =\alpha_{n} e_{2 n}, \quad n=1,2, \ldots, \\
S e_{2 n-1} & =\alpha_{n} e_{2 n-1}, \quad n=1,2, \ldots,
\end{aligned}
$$

for a bounded sequence $\left(\alpha_{n}\right)$ satisfies $\|S\| \leqslant C_{1} \sup \left|\alpha_{n}\right|$. (The constant $C_{1}$ is not 1 , since $\|\cdot\|$ is not a norm, but merely a quasi-norm). Thus, as remarked in [4], $Z_{2}$ has an unconditional Schauder decomposition into two-dimensional subspaces.

We shall use repeatedly the "symmetry" of this Schauder decomposition, particularly in arguments where we pass to a subsequence.

Let $\left(w^{(n)}: n=1,2, \ldots\right)$ be a bounded block basic sequence (with respect to $\left(e_{n}\right)$ ) contained in $M$. We shall define the induced block basic operator $W: Z_{2} \rightarrow Z_{2}$ by

$$
\begin{array}{ll}
W e_{2 n-1}=w^{(n)}, & n=1,2, \ldots, \\
W e_{2 n}=\phi\left(v^{(n)}\right), & n=1,2, \ldots,
\end{array}
$$

where $v^{(n)} \in l_{2}$ is given by $v_{k}^{(n)}=w_{2 k-1}^{(n)}$. To show that $W$ is bounded we first check that if $\left\|w^{(n)}\right\|=1$ for every $n$, then $W$ is an isometry. Suppose that

$$
w^{(n)}=\sum_{i=p_{n-1}+1}^{p_{n}} a_{i} e_{2 i-1},
$$

where $0=p_{0}<p_{1}<p_{2}<\cdots$; then $\sum_{p_{n-1}+1}^{p_{n}} a_{i}^{2}=1$. If $x \in \mathbf{R}^{\infty}$ and $E x=0$, then $\|W x\|=\|x\|$. Assume then that $\|E x\|_{2}=1$. If $p_{n-1}+1 \leqslant i \leqslant p_{n}$, and $W x=w$, then

$$
w_{2 i}=x_{2 n} a_{i}, \quad w_{2 i-1}=x_{2 n-1} a_{i}+x_{2 n} a_{i} \log 1 /\left|a_{i}\right| .
$$

Thus, $\Sigma w_{2 i}^{2}=\sum x_{2 n}^{2}=1$, and

$$
(\phi(E w))_{2 i-1}=x_{2 n} a_{i} \log \frac{1}{\left|x_{2 n} a_{i}\right|} .
$$


Now,

$$
\|w-\phi(E w)\|_{2}^{2}=\sum_{i=1}^{\infty}\left(x_{2 n-1}-x_{2 n} \log \frac{1}{\left|x_{2 n}\right|}\right)^{2} a_{i}^{2}=\|x-\phi(E x)\|_{2}^{2} .
$$

Therefore, if $\left\|w^{(n)}\right\|=1$ for all $n, W$ is an isometry. In general, if $w^{(n)}=\alpha_{n} v^{(n)}$ for $\left\|v^{(n)}\right\|=1$, then $W=V S$ where $V$ is the block basic operator given by $S e_{2 n}=\alpha_{n} e_{2 n}$, $S e_{2 n-1}=\alpha_{n} e_{2 n-1}$ discussed above. Hence, in general

$$
\|W\| \leqslant C_{1} \sup \left\|w^{(n)}\right\| .
$$

The algebra $\mathfrak{L}\left(Z_{2}\right)$ of all bounded linear operators on $Z_{2}$ is a ${ }^{*}$-algebra if we introduce the involution ${ }^{*}$ by

$$
\Omega(T x, y)=\Omega\left(x, T^{*} y\right), \quad x, y \in Z_{2} .
$$

Note that if $W$ is a block basic operator for $w^{(n)}$, where $\left\|w^{(n)}\right\|=1$ for all $n$, then

$$
\Omega(W x, W Y)=\Omega(x, y), \quad x, y \in Z_{2},
$$

(check this for basis elements) and hence $W^{*} W=I$.

Our next lemma is established in [6] and can be deduced from elementary calculations with block basic sequences.

LEMMA 3. Let $\left(u^{(n)}\right)$ be a basic sequence in $Z_{2}$ with $0<\inf \left\|u^{(n)}\right\| \leqslant \sup \left\|u^{(n)}\right\|<\infty$. Then:

(a) If $\lim _{n \rightarrow \infty}\left\|E u^{(n)}\right\|_{2}=0$ then $u^{(n)}$ has a subsequence equivalent to the usual basis of $l_{2}$.

(b) If lim $\sup _{n \rightarrow \infty}\left\|E u^{(n)}\right\|_{2}>0$ then $u^{(n)}$ has a subsequence equivalent to the usual basis of the Orlicz sequence space $l_{f}$ where $f$ is defined in a neighborhood of 0 by $f(t)=(t \log 1 / t)^{2}$.

REMARK. The statement of $3(\mathrm{~b})$ simply means that $u^{(n)}$ has a subsequence $v^{(n)}$ such that $\Sigma t_{n} v^{(n)}$ converges if and only if

$$
\sum_{n=1}^{\infty} t_{n}^{2}+\sum_{n=1}^{\infty}\left(t_{n} \log \frac{1}{t_{n}}\right)^{2}<\infty .
$$

The next lemma is a consequence of Lemma 3; see Theorem 6.4 of [4].

LEMMA 4. The quotient map $E: Z_{2} \rightarrow l_{2}$ is strictly singular.

LeMma 5. (a) If $T \in \mathcal{L}\left(Z_{2}\right)$, then

$$
\lim _{n \rightarrow \infty}\left\|E T e_{2 n-1}\right\|_{2}=0 .
$$

(b) If $T \in \mathscr{L}\left(Z_{2}\right)$ is strictly singular then

$$
\lim _{n \rightarrow \infty}\left\|E T e_{2 n}\right\|_{2}=0 \text {. }
$$

Proof. (a) $E T: M \rightarrow l_{2}$ is strictly singular and hence as $M \cong l_{2}, E T$ is compact (see [2]).

(b) Otherwise there is a subsequence of $\left(T e_{2 n}\right)$ which is a basic sequence equivalent to $\left(e_{2 n}\right)$ by Lemma 3(b) and so $T$ is not strictly singular. 
Now define $L: Z_{2} \rightarrow Z_{2}$ by $L e_{2 n}=e_{2 n-1}$ and $L e_{2 n-1}=0$ for $n=1,2,3, \ldots$ Thus $\|L x\|=\|E x\|_{2}$ for $x \in Z_{2}$, and $L$ is strictly singular.

LEMMA 6. For $T \in \mathcal{L}\left(Z_{2}\right)$,

$$
\lim _{n \rightarrow \infty}\left\|L T e_{2 n}-T e_{2 n-1}\right\|=0 .
$$

Proof. By passing to subsequences it suffices to consider the case when

$$
\sum_{n=1}^{\infty}\left\|T e_{2 n-1}-w^{(n)}\right\|<\infty
$$

for a bounded block basic sequence $w^{(n)}$ in $M$; note $d\left(T e_{2 n-1}, M\right) \rightarrow 0$ by Lemma $5(\mathrm{a})$.

Let $W$ be the associated block basic operator. Define $K: Z_{2} \rightarrow Z_{2}$ by

$$
\begin{gathered}
K e_{2 n-1}=T e_{2 n-1}-w^{(n)}, \quad n=1,2, \ldots, \\
K e_{2 n}=0, \quad n=1,2, \ldots
\end{gathered}
$$

Then $K$ is compact and $[T-(W+K)](M)=(0)$. Hence $T-(W+K)$ factors through $E$ and is strictly singular. Thus $T-W$ is also strictly singular and so $\left\|E(T-W) e_{2 n}\right\|_{2} \rightarrow 0$, i.e.

$$
\left\|L(T-W) e_{2 n}\right\| \rightarrow 0
$$

However $L W e_{2 n}=w^{(n)}$.

Now let $\mathcal{U}$ be a nonprincipal ultrafilter on $\mathbf{N}$. Define a bilinear form on $\mathfrak{L}\left(Z_{2}\right)$ by

$$
(S, T)=\lim _{n \in \mathcal{Q}} \Omega\left(S e_{2 n-1}, T e_{2 n}\right) \text {. }
$$

Denote by $\langle$,$\rangle the standard inner product on l_{2}$.

LEMMA 7. (a) $(S, T)=\lim _{n \in \mathcal{Q}}\left\langle E S e_{2 n}, E T e_{2 n}\right\rangle$.

(b) $(T, T)=\lim _{n \in \mathcal{Q}}\left\|T e_{2 n-1}\right\|^{2}$.

Proof. (a) By Lemma 6,

$$
\lim _{n \in \mathcal{Q}} \Omega\left(S e_{2 n-1}, T e_{2 n}\right)=\lim _{n \in \mathcal{Q}} \Omega\left(L S e_{2 n}, T e_{2 n}\right)=\lim _{n \in \mathscr{Q}}\left\langle E S e_{2 n}, E T e_{2 n}\right\rangle .
$$

(b) $(T, T)=\lim _{n \in \mathcal{Q}}\left\|E T e_{2 n}\right\|^{2}=\lim _{n \in \mathcal{Q}}\left\|T e_{2 n-1}\right\|^{2}$ by Lemma 6 .

Lemma 7 shows that $($,$) is a nontrivial symmetric nonnegative bilinear form on$ $\mathcal{L}\left(Z_{2}\right)$. Let $H$ be the Hilbert space obtained by completing the quotient of $\mathcal{L}\left(Z_{2}\right)$ by the space $(T:(T, T)=0)$. Then there is an algebra homomorphism $\Lambda: \mathcal{L}\left(Z_{2}\right) \rightarrow \mathcal{L}(H)$ defined by $\Lambda(A) T=A T, A, T \in \mathcal{L}\left(Z_{2}\right)$.

Note by Lemma $7(\mathrm{~b})$ that $\|\Lambda(A)\| \leqslant\|A\|$.

If $A \in \mathcal{L}\left(Z_{2}\right)$ then

$$
\begin{aligned}
\left(\Lambda\left(A^{*}\right) S, T\right) & =\lim _{\mathscr{Q}} \Omega\left(A^{*} S e_{2 n-1}, T e_{2 n}\right)=\lim _{\mathscr{Q}} \Omega\left(S e_{2 n-1}, A T e_{2 n}\right) \\
& =(S, \Lambda(A) T) .
\end{aligned}
$$

Thus $\Lambda\left(A^{*}\right)=\Lambda(A)^{*}$ and $\Lambda$ is a ${ }^{*}$-homomorphism. Clearly $\Lambda(I)=I$ and so $\Lambda$ is nontrivial. Denote by $\mathscr{G}$ the ${ }^{*}$-ideal $(T: \Lambda(T)=0)$.

TheOREM 8. $Z_{2}$ contains no Lagrangian subspace. 
Proof. If $Z_{2}$ has a Lagrangian subspace, there is a projection $P$ so that

$$
\begin{gathered}
\Omega(P x, P y)=0, \quad x, y \in Z_{2}, \\
\Omega((I-P) x,(I-P) y)=0, \quad x, y \in Z_{2} .
\end{gathered}
$$

Now $P^{*} P=0$ and so $\Lambda\left(P^{*} P\right)=0$. Hence $\Lambda(P)^{*} \Lambda(P)=0$ and thus $\Lambda(P)=0$. Similarly $\Lambda(I-P)=0$ and thus $\Lambda(I)=0$ which is a contradiction.

We can extend Theorem 8 a little by identifying the kernel of the homomorphism $\Lambda$.

LEMMA 9. 9 is the ideal of strictly singular operators in $\mathcal{L}\left(Z_{2}\right)$ and this ideal is a maximal two-sided ideal.

Proof. If $A \in \mathcal{L}\left(Z_{2}\right)$ is strictly singular then Lemma $5(\mathrm{~b})$ implies $\Lambda(A)=0$. For the converse use the argument in Theorem 6.5 of [6] to show that if $A$ is not strictly singular then there are invertible operators $U_{1}, U_{2}$ and block basic operators $W, V$ so that $W=U_{1} A U_{2} V$, and $W$ corresponds to block basic sequence $w^{(n)}$ with $\left\|w^{(n)}\right\|=1$. Then $W^{*} W=I$ and so if $A$ belongs to some two-sided ideal, this ideal is trivial.

RemarK. This argument also can be used to show that the range of $W$ is complemented, which was first observed by Johnson, Lindenstrauss and Schectman [5].

THEOREM 10. If $F$ is a complemented isotropic subspace of $Z_{2}$ then $\operatorname{dim} F<\infty$.

Proof. If $P$ is a projection on $F$, then $\Lambda(P)=0$.

Remark. We do not know if $\Lambda$ maps onto a closed ${ }^{*}$-subalgebra of $\mathscr{B}(H)$. Clearly this example can be "complexified" to yield, for complex $Z_{2}$, that $\varrho\left(Z_{2}\right)$ is a *-algebra whose strong radical [1] is the ideal 9 of strictly singular operators. The question is then whether $\mathcal{L}\left(Z_{2}\right) / \mathscr{G}$ is *-isomorphic to a $B^{*}$-algebra.

\section{REFERENCES}

1. F. F. Bonsall and J. Duncan, Complete normed algebras, Ergebnisse des Mathematik, Band 80, Springer-Verlag, New York, Heidelberg and Berlin, 1973.

2. P. Chernoff and J. Marsden, Properties of infinite dimensional Hamiltonian systems, Lecture Notes in Math., vol. 425, Springer-Verlag, Berlin and New York, 1974.

3. J. Duistermaat, On the Morse index theorem in variational calculus, Adv. in Math. 21 (1976), $173-195$.

4. R. H. Herman, On the uniqueness of the ideals of compact and strictly singular operators, Studia Math. 29 (1968), 161-165.

5. W. B. Johnson, J. Lindenstrauss and G. Schechtman, On the relation between several notions of unconditional structure, Israel J. Math. 37 (1980), 120-129.

6. N. J. Kalton and N. T. Peck, Twisted sums of sequence spaces and the three space problems, Trans. Amer. Math. Soc. 255 (1979), 1-30.

7. E. C. Lance, Quadratic forms on Banach spaces, Proc. London Math. Soc. (3) 25 (1972), 341-357.

8. R. C. Swanson, Linear symplectic structures on Banach spaces, Rocky Mountain J. Math. 10 (1980), 305-317.

9.___ Fredholm intersection theory and elliptic boundary deformation problems. I and II, J. Differential Equations 28 (1978), 189-219.

10. A. Weinstein, Symplectic manifolds and their Lagrangian submanifolds, Adv. in Math. 6 (1971), $329-346$.

Department of Mathematics, University of Missouri, Columbia, Missouri 65211 